\title{
Improved Infrared Multiphoton Dissociation of Peptides through N-Terminal Phosphonite Derivatization
}

\author{
Lisa A. Vasicek, Jeffrey J. Wilson, and Jennifer S. Brodbelt \\ Department of Chemistry and Biochemistry, The University of Texas at Austin, Austin, Texas, USA
}

\begin{abstract}
A strategy for improving the sequencing of peptides by infrared multiphoton dissociation (IRMPD) in a linear ion trap mass spectrometer is described. We have developed an N-terminal derivatization reagent, 4-methylphosphonophenylisothiocyanate (PPITC), which allows the attachment of an IR-chromogenic phosphonite group to the N-terminus of peptides, thus enhancing their IRMPD efficiencies. After the facile derivatization process, the PPITCmodified peptides require shorter irradiation times for efficient IRMPD and yield extensive series of $y$ ions, including those of low $\mathrm{m} / \mathrm{z}$ that are not detected upon traditional CID. The resulting IRMPD mass spectra afford more complete sequence coverage for both model peptides and tryptic peptides from cytochrome $c$. We compare the effectiveness of this derivatization/IRMPD approach to that of a common N-terminal sulfonation reaction that utilizes 4-sulfophenylisothiocyanate (SPITC) in conjunction with CID and IRMPD. (J Am Soc Mass Spectrom 2009, 20, 377-384) (c) 2009 Published by Elsevier Inc. on behalf of American Society for Mass Spectrometry
\end{abstract}

$\mathrm{I}$ $\mathrm{n}$ recent years, there has been tremendous effort devoted to the application of mass spectrometry to the field of proteomics $[1,2]$ in large part because of the success of tandem mass spectrometry for elucidation of primary sequences and modifications of peptides and proteins [3-7]. Several ion activation methods have been developed in this context, including collision induced dissociation (CID) [8,9], electron capture dissociation (ECD) [10]. electron-transfer dissociation (ETD) [11], pulsed-Q dissociation (PQD) [12], surface induced dissociation [13], and photodissociation (PD) [14-19]. Each method has its own particular strengths and shortcomings, with CID being the most widely used due to its relatively well-understood underpinnings. However, the resulting CID mass spectra can be cluttered with redundant $b$ and $y$ ions, as well as ions created by uninformative losses of small organic molecules like water and ammonia. Hence, the interpretation of such data manually or via de novo algorithms remains challenging [20, 21]. ECD and ETD provide complementary $c$ and $z$ ions and have proven more useful for tracking post-translational modifications (PTMs); however, these activation methods generally offer lower dissociation efficiencies than CID and are highly dependent on the charge state of the selected precursor ion [10, 11]. PD methods, including both infrared multiphoton dissociation (IRMPD) and ultraviolet photodissociation (UVPD), have also shown

Address reprint requests to Dr. J. S. Brodbelt, Department of Chemistry and Biochemistry, The University of Texas at Austin, 1 University Station A5300, Austin, TX 78712, USA. E-mail: jbrodbelt@mail.utexas.edu promise because of their potential for high-energy deposition and tunability [14-19, 22-35]. Since photoabsorption is not a collision-based process, it does not alter the kinetic energies of ions and thus minimizes any ion losses due to scattering.

Photodissociation also offers particular advantages for ion trap mass spectrometers. For example, photoactivation is independent of the trapping voltage, unlike CID. In CID, the rf voltage during ion activation influences the energy deposition of collisional activation as well as defines the lower $m / z$ range. The potential for greater energy deposition in CID occurs at the expense of storage of lower $\mathrm{m} / \mathrm{z}$ ions, ones which might be key diagnostic ions for sequencing peptides. PQD is another collision based activation method used as an alternative for alleviating the limited $\mathrm{m} / \mathrm{z}$ storage problem during CID in ion trap mass spectrometers. For PQD, the rf voltages applied during ion activation and subsequent ion trapping are quickly manipulated to avoid ejection of all the lower $m / z$ product ions [12]. However, the dissociation efficiency for PQD, in terms of the conversion of precursor ions to product ions, is rather low. Despite this impressive array of ion activation methods, the quest for more efficient and more selective strategies continues to be an important goal in the development of mass spectrometric-based proteomics, especially to simplify spectral interpretation and improve the identification of post-translational modifications.

One approach entails derivatization of proteins or peptides to facilitate their enrichment in complex mixtures, [36-39] to alter their charge states to direct fragmentation pathways, [16] or to attach chromo-
(C) 2009 Published by Elsevier Inc. on behalf of American Society for Mass Spectrometry. 1044-0305/09/\$32.00

doi:10.1016/j.jasms.2008.10.016
Published online October 30, 2008 Received August 14, 2008 Revised October 24, 2008 Accepted October 27, 2008 
phores to enhance their photoabsorptivity [14-16, 29, $32,40,41]$. For example, N-terminal sulfonation reactions have proven to simplify the MS/MS spectra of peptides by introducing a fixed negative charge at the N-terminus [42-45]. This sulfonate addition leads to neutralization of the normally singly charged $b$ ions created upon dissociation of peptides, resulting in series of easily interpreted $y$ ions. In particular, 4sulfophenylisothiocyanate (SPITC) has shown great potential as a reagent for this process because it is inexpensive, stable, and extremely efficient in reacting with the N-terminus of peptides [43, 45, 46]. Upon CID, the dominant product ion for the SPITC-derivatized peptides is typically the $y_{n-1}$ ion due to the loss of the sulfanilic acid and the N-terminal amino acid which is catalyzed by the labile proton from the sulfonic group that promotes a specific Edman-type cleavage [43]. Another N-terminal sulfonation reagent, 2-sulfobenzoic acid cyclic anhydride, also introduces a negative charge at the N-termini of peptides, but the resulting peptides dissociate to give a broader array of $y$ ions than afforded by the SPITC-derivatized peptides [16]. The newest $\mathrm{N}$-terminal sulfonation reagent, 4-chlorosulfophenyl isocyanate, also results in more uniform arrays of y ions from the peptides, albeit with higher critical energies [46]. PITC is another N-terminal reagent that affords a good chromophore at $266 \mathrm{~nm}$ for UVPD, and the resulting PITC-peptides yield both $b$ and $y$ sequence ions [47]. The same SPITC-derivatized peptides produce only y ions upon UVPD, again with notable enhancement of the $y_{n-1}$ ion [47].

We have been developing derivatization agents for biological molecules that increase their photoabsorptivities in the gas phase and/or alter the fragmentation patterns of the resulting ions to give more informative or more easily interpreted mass spectra. For example, we recently combined an N-terminal sulfonation procedure with IRMPD in a quadrupole ion trap to allow detection of entire series of y ions for tryptic peptides, thus allowing successful de novo sequencing [16]. We have also developed other IR-chromogenic ligands to improve IRMPD efficiencies, including phosphorylated pyridyl ligands that form metal complexes with flavonoids [41], a boronic acid reagent that selectively binds to the diol functionality of oligosaccharides and improves the determination of their sequences [40], and a new phosphorylated crosslinking reagent that allows the differentiation of crosslinked peptides from noncrosslinked peptides for elucidation of contact points in proteins [48]. We have also explored UV-chromogenic reagents in conjunction with UVPD for sequencing peptides [15] and a reductive amination procedure that incorporates fluorescent labels into oligosaccharides to improve UVPD efficiencies [14]. The successful outcomes of these previous strategies that combined derivatization with photodissociation have motivated our efforts to expand the arsenal of targeted chromogenic reagents for tunable photodissociation of biological molecules.
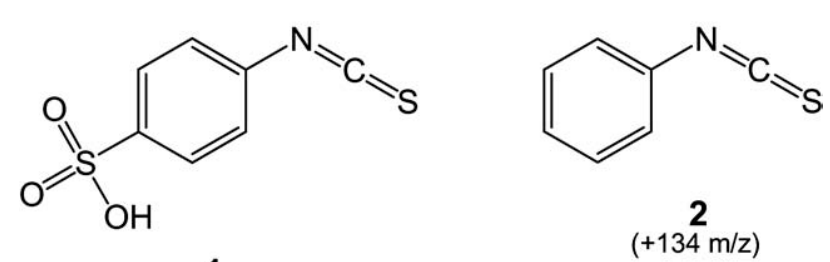

$(+215 \mathrm{~m} / \mathrm{z})$

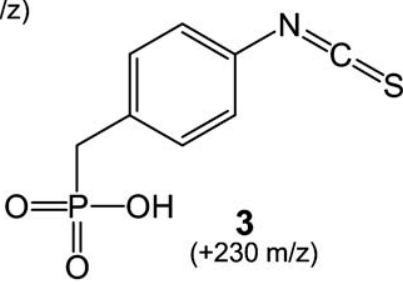

Figure 1. PITC compounds; (1) 4-sulfophenylisothiocyanate (SPITC), (2) phenylisothiocyanate (PITC), (3) 4-methylphosphonophenylisothiocyanate (PPITC)

In the present study, we report a new N-terminal derivatization reagent that incorporates a phosphonite functionality. The new reagent, 4-methylphosphonophenylisothiocyanate (PPITC), was designed specifically to provide a negative charge site similar to the sulfonated reagents, in addition to containing a strong IR chromophore to enhance the photoabsorptivity at a wavelength of $10.6 \mu \mathrm{m}$ of the resulting derivatized peptides. It has been shown previously that phosphorylated peptides possess high photoabsorptivities at 10.6 $\mu \mathrm{m}$, which is critical for successful IRMPD [23, 49-51]. Here we compare the IRMPD efficiencies and the number of diagnostic sequence ions obtained for a series of peptides derivatized by SPITC, PPITC, and PITC (Figure 1).

\section{Experimental}

\section{Reagents}

All chemicals were purchased from Sigma Aldrich (St. Louis, MO) except the following peptides, which were obtained from BACHEM (King of Prussia, PA): LQVQLSIR (H-4588), FSWGAEGQR (H-2680), Amyloid protein 1-16, and YGGFLK (H-2765); and from American Peptide Co. (Sunnyvale, CA): HSDAVFTDNYTR (48-1-12) and Fibrinopeptide A (42-1-12A).

\section{Synthesis of 4-Methylphosphonophenylisothiocyanate (3)}

4-Bromophenylisothiocyanate was refluxed for $30 \mathrm{~min}$ with $2 \times$ triethylphosphite, after which the product was purified on a silica column with 50:50 DCM:MeOH and dried to isolate the diethyl ester form. The ester form was converted to the acidic form by reaction with 3.5 equivalents of bromotrimethylsilane, which was added drop-wise and allowed to stir at room temperature for $24 \mathrm{~h}$. The reaction mixture was dried on a rotovap, and the product was dissolved in minimal $\mathrm{MeOH}$. This product was allowed to stir at room temperature for an additional 4 hours and then dried on a rotovap and 
concentrated under reduced pressure. The PPITC structure was confirmed by ${ }^{1} \mathrm{H}$ NMR using a $400 \mathrm{MHz}$ Varian instrument (Varian, Palo Alto, CA). ${ }^{1} \mathrm{H}$ NMR at $400 \mathrm{MHz}$ in $\mathrm{CD}_{3} \mathrm{OD}$ yielded: $7.21 \mathrm{ppm}(\mathrm{d}, 2 \mathrm{H}), 7.40 \mathrm{ppm}$ $(\mathrm{d}, 2 \mathrm{H}), 3.17 \mathrm{ppm}(\mathrm{s}, 2 \mathrm{H}), 3.19 \mathrm{ppm}(\mathrm{s}, 1 \mathrm{H})$; these findings are consistent with the proposed structure.

\section{PITC Derivatization of Peptides}

PITC and SPITC protocol: $20 \mu \mathrm{L}$ of a $10 \mu \mathrm{g} / \mu \mathrm{L}$ solution of reagent solution buffered to a $\mathrm{pH}$ of 9.5 with $20 \mathrm{mM}$ $\mathrm{NaHCO}_{3}$ was incubated with $10 \mu \mathrm{L}$ of $1 \mathrm{mM}$ peptide solution for $30 \mathrm{~min}$ at $55^{\circ} \mathrm{C}$. The reaction was terminated with an addition of $1.5 \mu \mathrm{L}$ of $5 \%$ trifluoroacetic acid, and then derivatized product was purified on a C18 spin column.

PPITC protocol: $20 \mu \mathrm{L}$ of a $10 \mu \mathrm{g} / \mu \mathrm{L}$ solution of reagent solution buffered to a $\mathrm{pH}$ of 9.5 with $20 \mathrm{mM}$ $\mathrm{NaHCO}_{3}$ was incubated with $10 \mu \mathrm{L}$ of $1 \mathrm{mM}$ peptide solution, $60 \mu \mathrm{L} 100 \mathrm{mM} \mathrm{NaHCO}_{3}(\mathrm{pH} 8-9)$ and $70 \mu \mathrm{L}$ water for $30 \mathrm{~min}$ at $55^{\circ} \mathrm{C}$. The product was purified on a C18 spin column and diluted to $10 \mathrm{uM}$ before ESI analysis.

\section{Mass Spectrometry}

All experiments were performed on a ThermoFinnigan LTQ XL linear ion trap mass spectrometer (ThermoFinnigan, Waltham, MA) equipped with a standard ESI source. The LTQ mass spectrometer was modified for IRMPD as described earlier [48]. Solutions were directly infused at a flow rate of $3 \mu \mathrm{L} / \mathrm{min}$ at a concentration of $10 \mu \mathrm{M}$ in $100 \% \mathrm{MeOH}$. For CID, the precursor was activated for $30 \mathrm{~ms}$ at a standard $q_{z}$ value of 0.25. Energy-variable CID experiments were performed by incrementally raising the applied CID voltage. All CID experiments were recorded in triplicate on the same day. In cases where CID voltages are reported, the CID voltages required to cause dissociation of $50 \%$ of the selected precursor ions were corrected for their number of degrees of freedom using the following equation:

$$
E_{(50 \% \text { corrected })}=E_{(50 \%)} \times \frac{N_{u \text { modified }}}{N_{\text {derivatized }}}
$$

where the degrees of freedom is defined as $N=3 n-6$, and $n$ is the number of atoms in the underivatized peptide [52].

\section{Infrared Multiphoton Dissociation}

IRMPD experiments were performed using a 48-5 Synrad 50-W continuous wave CO2 laser (Mukilteo, WA). The laser was directed through the back end of the vacuum manifold that was modified with a $C F$ view port flange equipped with a $\mathrm{ZnSe}$ window to allow the $10.6 \mu \mathrm{m}$ radiation into the ion trap [48]. This port was aligned so that the beam was on axis with a $1.7 \mathrm{~mm}$ entrance aperture and the $2 \mathrm{~mm}$ aperture of the exit lens of the ion trap. The unfocused laser was controlled using a TTL signal setup in the instrument software to trigger during activation. All IRMPD experiments were performed using a reduced $q_{z}$ value of 0.1 to allow for sufficient detection of the low-mass ions and irradiation times of $\sim 5$ to $200 \mathrm{~ms}$ at $50 \mathrm{~W}$ unless otherwise noted. The helium pressure was maintained at the normal operating pressure. Time-variable IRMPD experiments were executed by incrementally increasing the irradiation time.

\section{Results and Discussion}

\section{Design and Reactions of PPITC}

We have previously shown that combining IRMPD with an N-terminal sulfonation procedure substantially improved the de novo sequencing of peptides, primarily by eliminating the redundant $b$ ion series that can make it difficult to pinpoint the $y$ ions and also by extending the series of $y$ ions detected via alleviation of the low mass cut-off typically encountered upon conventional CID [16]. The promising results obtained in this prior study motivated us to design a new $\mathrm{N}$ terminal derivatization reagent that would specifically contain an IR chromophore to enhance the absorption of $10.6 \mu \mathrm{m}$ photons, thus making the peptides even more amenable to IRMPD. In addition, the new reagent was designed to afford a fixed negative charge site at the N-terminus to encourage the neutralization of all $b$ products, yielding $y$-rich fragmentation patterns similar to what has been observed previously following derivatization of peptides with SPITC [43, 45, 46]. The presence of the negatively charged phosphonite group fixed at the $\mathrm{N}$-terminus neutralizes the conventional $\mathrm{N}$-terminal product ions (i.e., typically $b$ ions) created upon ion activation, resulting in detection of solely the C-terminal ions (i.e., typically y ions). The phosphonoPITC reagent shown in Figure 1 resulted in the attainment of both goals. The phosphonite group was incorporated on the PITC skeleton via a two-step modification of 4-bromophenylisothiocyanate. Subsequent reaction of PPITC with individual peptides or mixtures of tryptic peptides occurred with yields of $40 \%$ to $90 \%$, thus resulting in formation of ample PPITCderivatized peptides for IRMPD. In comparison, the average yields for derivatization of the same peptides using PITC or SPITC were typically $20 \%$ to $40 \%$ and 70 to $90 \%$, respectively.

\section{CID and IRMPD of PITC-Derivatized Peptides}

Examples of the CID and IRMPD mass spectra obtained for the underivatized and the corresponding PPITCderivatized peptides are shown in Supplemental Figure 1 and Figure 2, which can be found in the electronic version of this article. The CID mass spectrum of the first protonated peptide, FSWGAEGQR, is dominated 
by two product ions due to dehydration, and the diagnostic sequence ions have low relative abundances (Supplemental Figure 1A). Moreover, the spectrum is cluttered with both $b$ and $y$ ions that offer redundant and yet incomplete sequence information, in addition to additional confounding product ions attributed to ammonia or water losses. IRMPD of the underivatized peptide is very inefficient, resulting in no dissociation (spectrum not shown). Following the derivatization of this peptide with the PPITC reagent, IRMPD results exclusively in a complete series of $y$ ions from $y_{1}$ to $y_{8}$ (Supplemental Figure 1C). CID of the PPITC-peptide (Supplemental Figure 1B) results in the formation of the $y_{n-1}$ as the most abundant sequence ion (i.e., $y_{8}$ ), a finding similar to what has been previously reported for CID of SPITC-derivatized peptides [46, 53, 54]. CID or IRMPD of the modified peptide also results in the formation of the $[\mathrm{M}+\mathrm{CS}+\mathrm{H}]^{+}$product stemming from loss of part of the PPITC moiety $(-188 \mathrm{Da})$. This characteristic fragmentation pathway results in an easily tracked product ion that allows ready differentiation of derivatized and nonderivatized peptides that might be present in mixtures.

The CID and IRMPD mass spectra obtained for a larger peptide, fibrinopeptide A (ADSGEGDFLAEGGGVR), are shown in Figure 2. The CID mass spectrum of the unmodified protonated peptide (Figure 2a) is dominated by two $y$ ions $\left(y_{9}\right.$ and $\left.y_{14}\right)$ in addition to a few other $y$ ions of low abundance, dehydrated $y$ ions, and internal product ions of low abundance. This spectrum is unsatisfactory for de novo sequencing or manual interpretation, especially if the primary protein sequence of origin is unknown before analysis. IRMPD of the unmodified protonated peptide again results in no dissociation (spectrum not shown), indicating that the peptide exhibits poor IR absorptivity and undergoes

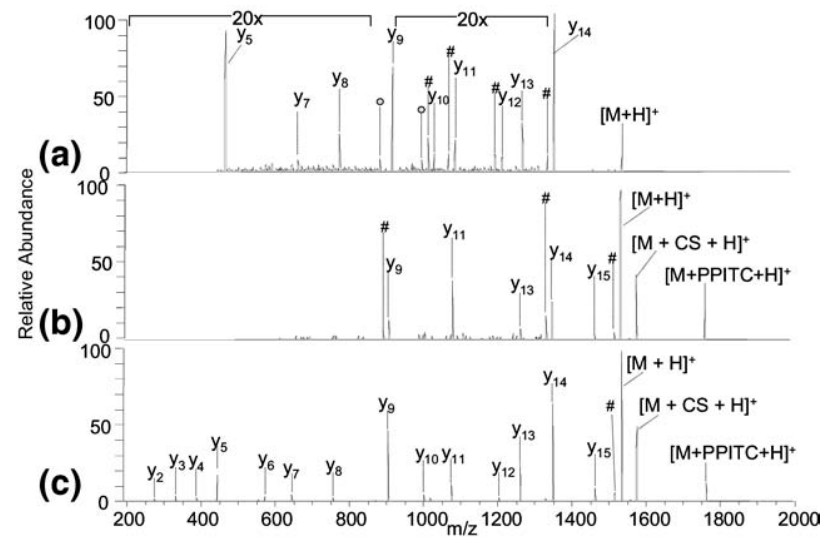

Figure 2. ESI MS / MS of protonated fibrinopeptide A; (a) CID of unmodified peptide (CID voltage $0.11 \mathrm{~V}, 30 \mathrm{~ms}$ ), fibrinopeptide A, (b) CID of PPITC-modified peptide (CID voltage $0.13 \mathrm{~V}, 30 \mathrm{~ms}$ ), (c) IRMPD of PPITC-modified peptide (laser power $50 \mathrm{~W}, 10 \mathrm{~ms}$ ). The $[\mathrm{M}+\mathrm{CS}+\mathrm{H}]^{+}$ion represents the partial loss of PPITC reagent. Water losses are represented by the pound symbol and internal products by an open circle. Magnification applies to all spectra over mass range indicated.

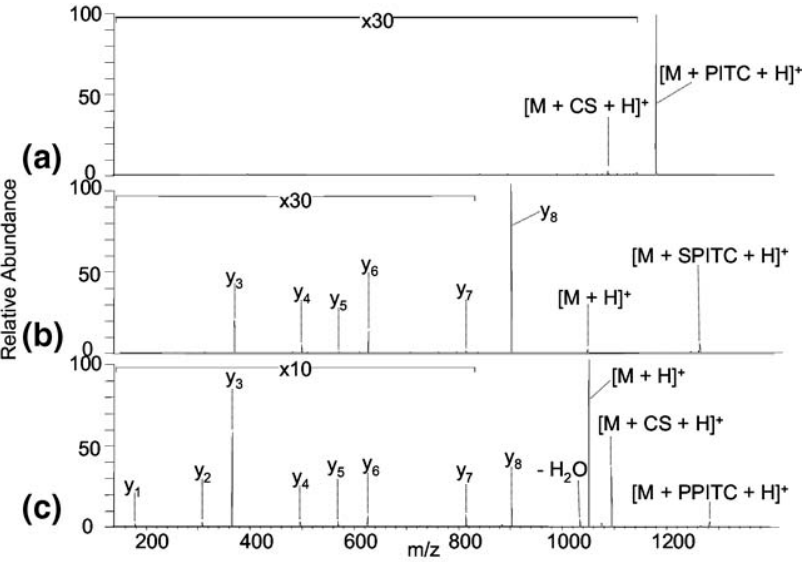

Figure 3. IRMPD of protonated derivatized FSWGAEGQR; (a) PITC-peptide (laser power $50 \mathrm{~W}, 500 \mathrm{~ms}$ ), (b) SPITC-modification (laser power $50 \mathrm{~W}, 25 \mathrm{~ms}$ ), (c) PPITC modification (laser power 50 $\mathrm{W}, 20 \mathrm{~ms})$. The $[\mathrm{M}+\mathrm{CS}+\mathrm{H}]^{+}$ion represents the partial loss of derivatization reagent.

collisional deactivation more efficiently than energization. CID of the PPITC-derivatized peptide results in the creation of different $y$ ions than detected for the underivatized peptide $\left(y_{9}, y_{11}, y_{13}, y_{14}, y_{15}\right)$ but leaves the sequence of the entire $C$-terminal side of the peptide inconclusive (Figure 2b). Moreover, gaps in the second half of the $y$ series could lead to ambiguities in identifying the amino acid sequence or the incorrect assignment of residues. On the other hand, the IRMPD mass spectrum of the PPITC-derivatized peptide displays an extensive series of $y$ ions ranging from $y_{2}$ to $y_{15}$ (Figure 2c), making sequence interpretation for this peptide straightforward. The high absorptivity of the derivatized peptide is reflected by the short irradiation time (10 $\mathrm{ms}$ ) needed to obtain the IRMPD spectrum shown in Figure 3c. The use of this short irradiation time also may provide a simple way to differentiate derivatized peptides from other compounds in mixtures based on monitoring the response (or lack of response) of the various species to IRMPD.

As shown by Muddiman et al. [49-51], phosphorylation greatly increases IR absorptivities of peptides and this finding is consistent with our results for the PPITCderivatized peptides. To further probe the relative absorptivities of PITC-derivatized peptides, we examined the IRMPD mass spectra of peptides modified by PITC, SPITC, and PPITC. Representative results are shown in Figure 3 for one peptide, FSWGAEGQR. The PITCderivatized peptide exhibits little dissociation even upon $500 \mathrm{~ms}$ of irradiation at $10.6 \mu \mathrm{m}$ (Figure 3a), similar to what was observed for nonderivatized peptides and giving a clear indication of the poor absorptivity of the PITC-peptides. IRMPD of the SPITCpeptide results in the dominant production of the $y_{n-1}$ ion (in this case $y_{8}$ ) after $25 \mathrm{~ms}$ of irradiation (Figure $3 \mathrm{~b}$ ). In contrast, the entire series of $y$ ions is observed in the IRMPD mass spectrum of the corresponding PPITCpeptide after $20 \mathrm{~ms}$ (Figure 3c). Both the SPITC- and 
PPITC-derivatized peptides appear to have comparable absorptivities, but the PPITC-peptide yields a more complete series of $y$ ions.

CID and IRMPD mass spectra were acquired in triplicate for a series of eight PITC-, SPITC-, PPITCderivatized peptides, as well as the underivatized peptides. The results are summarized in bar graph form in Figure 4 in terms of sequence coverage percentage, which is defined as the number of $y$ sequence ions detected relative to the total number of possible $y$ sequence ions ( $y_{1}$ to $\left.y_{n-1}\right)$, expressed as a percentage. For this comparison, a $y$ ion is considered to be detected in a CID or IRMPD mass spectrum if it has a total peak area of $1 \%$ or greater. A value of $100 \%$ for the sequence coverage percentage means that every possible $y$ ion $\left(y_{1}\right.$ to $\left.y_{n-1}\right)$ is detected.

In most cases, the highest sequence coverage percentages were associated with the PPITC-derivatized peptides in conjunction with IRMPD, suggesting that IRMPD of the PPITC-peptides affords the optimal strategy for identification of peptide sequences. The main exception to this trend occurs for multiply charged ions [i.e., doubly protonated VIP and multiply protonated amyloid peptide (DAEFRHDSGYEVHHEK) (data not shown)]. For the multiply protonated peptides, the extra mobile proton allows both $a / b$ and $y$ ions to remain charged in the MS/MS spectra and thus results in both a more elaborate array of potential $a / b$ and $y$ ions and overall lower abundance $y$ ions amongst a more cluttered spectra. For YGGFLK, the sequence coverage percentage obtained upon CID of the unmodified peptide is comparable to that obtained upon IRMPD of the PPITC-derivatized peptide. However, the CID mass spectrum contains both $b$ and $y$ ions, which complicates the spectral interpretation, whereas the IRMPD spectrum displays only $y$ ions.

\section{Energy-Variable CID and IRMPD}

We previously found that the improvement in IRMPD efficiency afforded by the $\mathrm{N}$-terminal sulfonation of
Table 1. IRMPD irradiation times and CID voltages required to cause $50 \%$ dissociation of unmodified and modifiedfibrinopeptide A (protonated). All IRMPD irradiation values are $\pm 1 \mathrm{~ms}$ and all CID values are $\pm 0.001 \mathrm{~V}$ based upon triplicate measurements

\begin{tabular}{lcc}
\hline \multicolumn{1}{c}{ Peptide } & IRMPD irradiation time $(\mathrm{ms})$ & CID voltage $(\mathrm{V})$ \\
\hline \hline Unmodified & $>1000$ & 0.104 \\
PITC & $>1000$ & 0.107 \\
SPITC & 11.5 & 0.109 \\
PPITC & 9.0 & 0.106 \\
\hline
\end{tabular}

peptides (using 2-sulfobenzoic acid) was due in part to a significant decrease in the critical energies of the peptides [16]. For the present study, the CID voltages and IRMPD irradiation times needed to cause dissociation of $50 \%$ of the parent ion population were determined and are summarized in Table 1 for one representative peptide, fibrinopeptide $\mathrm{A}$. The required CID voltages do not change significantly for the unmodified, PITC-, SPITC-, and PPITC-derivatized peptides, suggesting that there is no substantial change in the critical energies. In contrast, there is a notable change in the IRMPD irradiation times for the same series of peptides, with the SPITC- and PPITC-derivatized peptides requiring less than $25 \mathrm{~ms}$ whereas the underivatized and PITC-derivatized peptides never reaching the $50 \%$ dissociation level even after $1000 \mathrm{~ms}$ of irradiation time. The results suggest that the SPITC-and PPITC-derivatized peptides possess significantly amplified IR absorptivities which make them more amenable to IRMPD.

It is also informative to consider the distributions of product ions obtained for the PPITC-derivatized peptides as a function of the CID voltage or IRMPD irradiation time. Monitoring the product ion distributions allows the genealogies of ions to be indirectly evaluated. Maps of the product ion distributions are shown in Figure 5 for one representative peptide, PPITC-derivatized fibrinopeptide A. The CID data show that all of the $y$ ions evolve simultaneously, and their relative contributions do not change once the

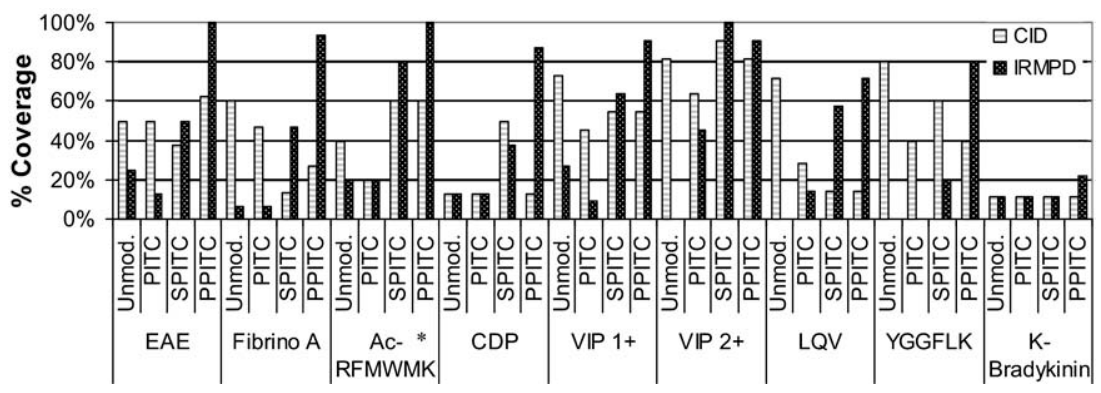

Figure 4. Sequence coverage percentages obtained by CID and IRMPD of unmodified and modified peptides, where the percent coverage is defined as the number of $y$ sequence ions detected relative to the total number of possible $y$ sequence ions $\left(y_{1}\right.$ to $\left.y_{n-1}\right)$, expressed as a percentage. *For AcRFMWMK, the percent coverage is defined as the number of $b$ sequence ions detected relative to the total number of possible $b$ sequence ions. Unmod. = unmodified peptide, EAE $=$ FSWGAEGQR, Fibrino $\mathrm{A}=$ ADSGEGDFLAEGGGVR, CDP $=$ CDPGYIGSR, VIP $=$ HSDAVFTDNYTR, LQV $=$ LQVQLSIR, $\mathrm{K}$-Brady $=$ KRPPGFSPFR. 


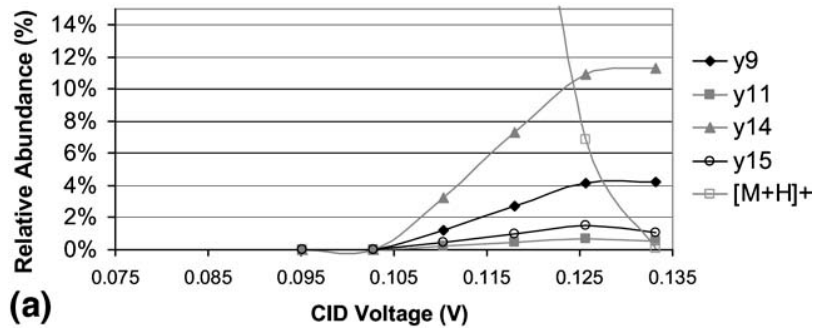

(a)

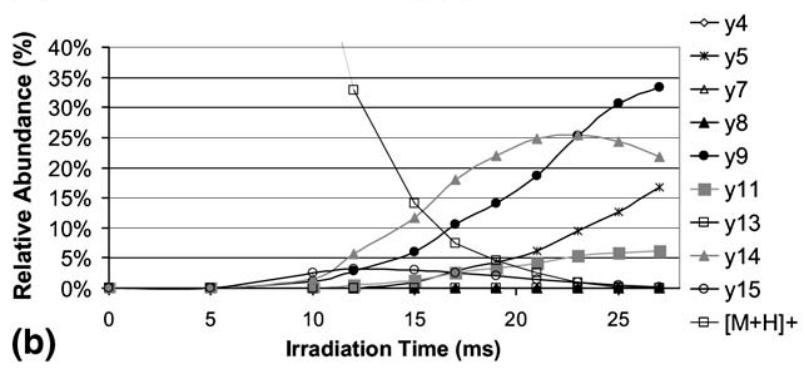

Figure 5. Energy-variable MS/MS distributions for $y$ ions of protonated PPITC-fibrinopeptide A and the surviving protonated precursor: (a) energy-variable CID and (b) time-variable IRMPD.

population of the protonated peptide is for the most part annihilated. The relative abundances of the various $y$ ions continue to change as the irradiation time is increased for the IRMPD data, even after the abundance of the parent ion is depleted. For example, the abundances of the $y_{4}$ and $y_{9}$ ions increase while the abundances of the $y_{14}$ and $y_{15}$ ions decrease at longer irradiation times. These complementary shifts in ion abundances support genealogical relationships between these sets of $y$ ions, and this reflects the non-resonant nature of IRMPD that promotes conversion of primary product ions to secondary product ions, thus leading to the greater array of diagnostic $y$ sequence ions.

\section{PPITC Derivatization and IRMPD of Tryptic Peptides}

The feasibility of the PPITC/IRMPD strategy was demonstrated for analysis of peptides obtained from the tryptic digestion of a protein, cytochrome $c$. The crude mixture of peptides obtained from enzymatic digestion of cytochrome $c$ was derivatized with PPITC as described earlier, and then subjected to C18 clean-up before ESI-MS/MS analysis via direct infusion. A typical ESI mass spectrum of the PPITC-derivatized tryptic digest is shown as Supplemental Figure 2. The majority of the peaks are assigned readily as underivatized peptides and their corresponding PPITC-derivatized analogs based on the characteristic mass shift associated with the PPITC modification. IRMPD, with an average irradiation time of $50 \mathrm{~ms}$ at $50 \mathrm{~W}$, and CID, with an average voltage of $0.58 \mathrm{~V}$ for $30 \mathrm{~ms}$, were used to analyze the PPITC-derivatized peptides, and the sequence coverage percentages are summarized in Figure 6. The sequence coverage percentages obtained by IRMPD are consistently higher or at least comparable to the values obtained by CID, thus demonstrating the feasibility of applying the PPITC/IRMPD approach for proteomics. For example, upon CID the PPITC-derivatized tryptic peptide MIFAGIK produced the $y_{3}, y_{4}$, and $y_{5}$ ions whereas the $y_{2}, y_{3}, y_{4}, y_{5}$, and $y_{6}$ ions were obtained upon IRMPD. For the sole tryptic peptide with a C-terminal arginine, an even greater increase in coverage was obtained by IRMPD. Following CID, only the $y_{5}$ and $y_{6}$ ions were observed for the $1^{+}$charge state while the $y_{2}, y_{3}$, and the $y_{5}-y_{10}$ were observed for the $2^{+}$ charge state. In comparison, IRMPD of the $1^{+}$peptide yielded the $y_{2}$ through $y_{7}$ ions, as well as the $y_{9}$ and $y_{10}$ ions, and IRMPD of the $2^{+}$peptide generated the $y_{2}, y_{3}$, $y_{5}, y_{6}, y_{9}$, and $y_{10}$ ions.

In general, the overall improvement in the sequence coverage percentages obtained upon IRMPD in comparison to CID of the PPTIC-derivatized tryptic peptides is not as great as was seen for the individual model peptides summarized in Figure 4, and this is attributed to several reasons. First, the relative complexity of the tryptic digest mixture leads to lower yields of PPITC-modified tryptic peptides and corresponding lower CID and IRMPD sensitivities. Second, the formation of predominantly lysine-terminated tryptic peptides in some cases leads to competitive formation of $a / b$ ions that divides the ion current among $a, b$, and $y$ ions and ultimately reduces the sensitivity of $y$ ion detection. This result may be attributed to the lower gas-phase basicity of lysine in comparison with arginine $[55,56]$. This difference in basicities of the terminal residues may change the proton mobility, thus altering the possibility of creating charged $a / b$ ions in comparison with the $y$ ions that stem from the sequestered $\mathrm{N}$ terminal proton. The sole arginine-terminated peptide, TGPNLHGLFGR, in the $1^{+}$charge state shows the greatest improvement in sequence coverage percentage upon IRMPD, thus supporting that the increased basicity of the arginine terminus assists in sequestering the proton on the C-terminus and thus eliminating the formation of charged $a / b$ ions. However, despite the decreased basicity of lysine-terminated tryptic peptides, IRMPD analysis following PPITC modification still afforded the highest sequence coverage compared with the other strategies (CID or using other derivatization reactions).

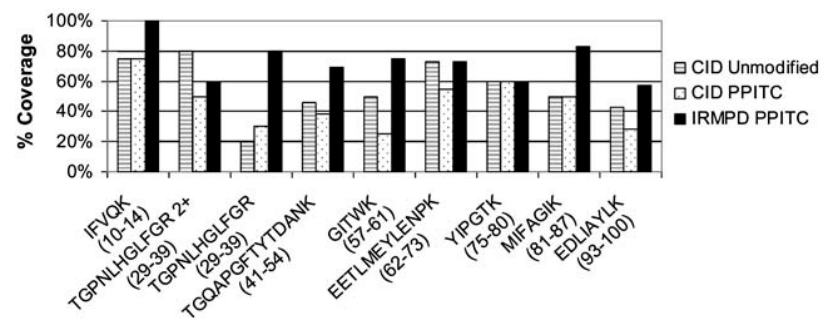

Figure 6. Sequence coverage percentages obtained by CID and IRMPD of unmodified and PPITC-modified tryptic peptides from cytochrome $c$, where the percent coverage is defined as the number of $y$ sequence ions detected relative to the total number of possible $y$ sequence ions $\left(y_{1}\right.$ to $\left.y_{n-1}\right)$, expressed as a percentage. 


\section{Conclusions}

N-terminal derivatization using a novel phosphorylated phenylisothiocyanate reagent in combination with IRMPD has been shown to be a promising approach for de novo sequencing of peptides. 4-Methylphosphonophenylisothiocyanate allows selective attachment of an IR chromophore to the peptides, thus enhancing their IR absorptivities and significantly reducing the irradiation times needed to cause efficient photodissociation. Extensive series of $y$ ions are created upon IRMPD of the PPITC-derivatized peptides, and the use of IRMPD allows detection of a greater array of the lower $\mathrm{m} / \mathrm{z} y$ ions than possible with conventional CID. The PPITC/ IRMPD strategy gives high sequence coverage percentages and has proven to be viable for analysis of tryptic peptides.

\section{Acknowledgments}

The authors gratefully acknowledge support from the Welch Foundation (F1155) and the National Science Foundation (CHE0718320).

\section{References}

1. Winston, R. L.; Fitzgerald, M. C. Mass Spectrometry as a Readout of Protein Structure and Function. Mass Spectrom. Rev. 1997, 16, 165-179.

2. Godovac-Zimmermann, J.; Brown, L. R. Perspectives for Mass Spectrometry and Functional Proteomics. Mass Spectrom. Rev. 2001, 20, 1-57.

3. Faux, M. C.; Scott, J. D. More on Target with Protein Phosphorylation: Conferring Specificity by Location. Trends Biochem. Sci. 1996, 21, 312315.

4. Lill, J. Proteomic Tools for Quantitation by Mass Spectrometry. Mass Spectrom. Rev. 2003, 22, 182-194.

5. Regnier, F. E.; Riggs, L.; Zhang, R.; Xiong, L.; Liu, P.; Chakraborty, A.; Seeley, E.; Sioma, C.; Thompson, R. A. Comparative Proteomics Based on Stable Isotope Labeling and Affinity Selection. J. Mass Spectrom. 2002, $37,133-145$

6. Sun, H.; Tonks, N. K. The Coordinated Action of Protein Tyrosine Phosphatases and Kinases in Cell Signaling. Trends Biochem. Sci. 1994, $19,480-485$.

7. Wysocki, V. H.; Resing, K. A.; Zhang, Q.; Cheng, G. Mass Spectrometry of Peptides and Proteins. Methods 2005, 35, 211-222.

8. Koy, C.; Mikkat, S.; Raptakis, E.; Sutton, C.; Resch, M.; Tanaka, K.; Glocker, M. O. Matrix-Assisted Laser Desorption/Ionization-Quadrupole Ion Trap Time of Flight Mass Spectrometry Sequencing Resolves Structures of Unidentified Peptides Obtained by In-Gel Tryptic Digestion of Haptoglobin Derivatives from Human Plasma Proteomes. Protion of Haptoglobin Deriv
teomics 2003, 3, 851-858.

9. Moyer, S. C.; Cotter, R. J.; Woods, A. S. Fragmentation of Phosphopeptides by Atmospheric Pressure MALDI and ESI/Ion Trap Mass Spectrometry. J. Am. Soc. Mass Spectrom. 2002, 13, 274-283.

10. Zubarev Roman, A. Electron-Capture Dissociation Tandem Mass Spectrometry. Curr. Opin. Biotechnol. 2004, 15, 12-16.

11. Syka, J. E. P.; Coon, J. J.; Schroeder, M. J.; Shabanowitz, J.; Hunt, D. F. Peptide and Protein Sequence Analysis by Electron Transfer Dissociation Mass Spectrometry. Proc. Natl. Acad. Sci. U.S.A. 2004, 101, 95289533.

12. Schwartz, J. C. High-Q Pulsed Fragmentation in Ion Traps. 2005, $20049416536949743 ; 20040914$

13. Dongre, A. R.; Somogyi, A.; Wysocki, V. H. Surface-Induced Dissociation: An Effective Tool to Probe Structure, Energetics, and Fragmentation Mechanisms of Protonated Peptides. J. Mass Spectrom. 1996, 31, $339-350$.

14. Wilson, J. J.; Brodbelt, J. S. Ultraviolet Photodissociation at $355 \mathrm{~nm}$ of Fluorescently Labeled Oligosaccharides. Anal. Chem. 2008, 80, 51865196.

15. Wilson, J. J.; Brodbelt, J. S. MS/MS Simplification by $355 \mathrm{~nm}$ Ultraviolet Photodissociation of Chromophore-Derivatized Peptides in a Quadrupole Ion Trap. Anal. Chem. 2007, 79, 7883-7892.

16. Wilson, J. J.; Brodbelt, J. S. Infrared Multiphoton Dissociation for Enhanced De Novo Sequence Interpretation of N-Terminal Sulfonated Peptides in a Quadrupole Ion Trap. Anal. Chem. 2006, 78, 6855-6862.

17. Payne, A. H.; Glish, G. L. Thermally Assisted Infrared Multiphoton Photodissociation in a Quadrupole Ion Trap. Anal. Chem. 2001, 73, 3542-3548.
18. Little, D. P.; Speir, J. P.; Senko, M. W.; O'Connor, P. B.; McLafferty, F. W Infrared Multiphoton Dissociation of Large Multiply Charged Ions for Biomolecule Sequencing. Anal. Chem. 1994, 66, 2809-2815.

19. Crowe, M. C.; Brodbelt, J. S. Infrared Multiphoton Dissociation (IRMPD) and Collisionally Activated Dissociation of Peptides in a Quadrupole Ion Trap with Selective IRMPD of Phosphopeptides. J. Am. Soc. Mass Spectrom. 2004, 15, 1581-1592.

20. Eng, J. K.; McCormack, A. L.; Yates, J. R. III. An Approach to Correlate Tandem Mass Spectral Data of Peptides with Amino Acid Sequences in a Protein Database. J. Am. Soc. Mass Spectrom. 1994, 5, 976-989.

21. Perkins, D. N.; Pappin, D. J. C.; Creasy, D. M.; Cottrell, J. S. ProbabilityBased Protein Identification by Searching Sequence Databases Using Mass Spectrometry Data. Electrophoresis 1999, 20, 3551-3567.

22. Colorado, A.; Shen, J. X.; Brodbelt, J. Use of Infrared Multiphoton Photodissociation with SWIFT for Electrospray Ionization and Laser Desorption Applications in a Quadrupole Ion Trap Mass Spectrometer. Anal. Chem. 1996, 68, 4033-4043.

23. Crowe, M. C.; Brodbelt, J. S. Differentiation of Phosphorylated and Unphosphorylated Peptides by High-Performance Liquid Chromatography-Electrospray Ionization-Infrared Multiphoton Dissociation in a Quadrupole Ion Trap. Anal. Chem. 2005, 77, 5726-5734.

24. Crowe, M. C.; Brodbelt, J. S.; Goolsby, B. J.; Hergenrother, P. Characterization of Erythromycin Analogs by Collisionally Activated Dissociation and Infrared Multiphoton Dissociation in a Quadrupole Ion Trap. J. Am. Soc. Mass Spectrom. 2002, 13, 630-649.

25. Goolsby, B. J.; Brodbelt, J. S. Tandem Infrared Multiphoton Dissociation and Collisionally Activated Dissociation Techniques in a Quadrupole Ion Trap. Anal. Chem. 2001, 73, 1270-1276.

26. Hashimoto, Y.; Hasegawa, H.; Yoshinari, K.; Waki, I. CollisionallyActivated Infrared Multiphoton Dissociation in a Quadrupole Ion Trap Mass Spectrometer. Anal. Chem. 2003, 75, 420-425.

27. Keller, K. M.; Brodbelt, J. S. Collisionally Activated Dissociation and Infrared Multiphoton Dissociation of Oligonucleotides in a Quadrupole Ion Trap. Anal. Biochem. 2004, 326, 200-210.

28. Shen, J.; Brodbelt, J. S. Characterization of Ionophore-Metal Complexes by Infrared Multiphoton Photodissociation and Collisionally Activated Dissociation in a Quadrupole Ion Trap Mass Spectrometer. Analyst 2000 $125,641-650$

29. Wilson, J. J.; Kirkovits, G. J.; Sessler, J. L.; Brodbelt, J. S. Photodissociation of Noncovalent Peptide-Crown Ether Complexes. J. Am. Soc. Mass Spectrom. 2008, 19, 257-260.

30. Devakumar, A.; Mechref, Y.; Kang, P.; Novotny, M. V.; Reilly, J. P. Identification of Isomeric N-Glycan Structures by Mass Spectrometry with $157 \mathrm{~nm}$ Laser-Induced Photofragmentation. J. Am. Soc. Mass Spectrom. 2008, 19, 1027-1040.

31. Thompson, M. S.; Cui, W.; Reilly, J. P. Mass Spectrometry: Fragmentation of Singly Charged Peptide Ions by Photodissociation at $1=157 \mathrm{~nm}$. Angew. Chem. Int. Ed. 2004, 43, 4791-4794.

32. Ly, T.; Julian, R. R. Residue-Specific Radical-Directed Dissociation of Whole Proteins in the Gas Phase. J. Am. Chem. Soc. 2008, 130, 351-358.

33. Yoon, S. H.; Chung, Y. J.; Kim, M. S. Time-Resolved Photodissociation of Singly Protonated Peptides with an Arginine at the N-Terminus: A Statistical Interpretation. J. Am. Soc. Mass Spectrom. 2008, 19, 645-655.

34. Moon, J. H.; Shin, Y. S.; Cha, H. J.; Kim, M. S. Photodissociation at 193 nm of Some Singly Protonated Peptides and Proteins with $\mathrm{m} / \mathrm{z} 2000$ 9000 Using a Tandem Time-of-Flight Mass Spectrometer Equipped with a Second Source for Delayed Extraction/Post-Acceleration of Product Ions. Rapid Commun. Mass Spectrom. 2007, 21, 359-368.

35. Yoon, S. H.; Kim, M. S. Development of a Time-Resolved Method for Photodissociation Mechanistic Study of Protonated Peptides: Use of a Voltage-Floated Cell in a Tandem Time-of-Flight Mass Spectrometer. J. Am. Soc. Mass Spectrom. 2007, 18, 1729-1739.

36. Krijgsveld, J.; Heck, A. J. R. Quantitative Proteomics by Metabolic Labeling with Stable Isotopes. Drug Discovery Today: Targets 2004, 3, S11-S15.

37. Qu, J.; Straubinger, R. M. Improved Sensitivity for Quantification of Proteins Using Triply Charged Cleavable Isotope-Coded Affinity Tag Peptides. Rapid Commun. Mass Spectrom. 2005, 19, 2857-2864.

38. Yomota, C.; Ohnishi, Y. Determination of Biotin Following Derivatization with 2-Nitrophenylhydrazine by High-Performance Liquid Chromatography with On-Line UV Detection and Electrospray-Ionization Mass Spectrometry. J. Chromatogr. A 2007, 1142, 231-235.

39. Li, H.; Schopfer, L.; Spaulding, R.; Thompson, C. M.; Lockridge, O. Identification of Organophosphate-Reactive Proteins by Tandem Mass Spectrometry. Chem. Biol. Interact. 2005, 157/158, 383-384.

40. Pikulski, M.; Hargrove, A.; Shabbir, S. H.; Anslyn, E. V.; Brodbelt, J. S Sequencing and Characterization of Oligosaccharides Using Infrared Multiphoton Dissociation and Boronic Acid Derivatization in a Quadrupole Ion Trap. J. Am. Soc. Mass Spectrom. 2007, 18, $2094-2106$.

41. Pikulski, M.; Wilson, J. J.; Aguilar, A.; Brodbelt, J. S. Amplification of Infrared Multiphoton Dissociation Efficiency in a Quadruple Ion Trap Using IR-Active Ligands. Anal. Chem. 2006, 78, 8512-8517.

42. Keough, T.; Youngquist, R. S.; Lacy, M. P. A Method for HighSensitivity Peptide Sequencing Using Post-Source Decay Matrix-Assisted Laser Desorption Ionization. Proc. Natl. Acad. Sci. U.S.A. 1999, 96, 7131-7136.

43. Lee, Y. H.; Shin, J.-W.; Ryu, S.; Lee, S.-W.; Lee, C. H.; Lee, K. Enrichment of N-Terminal Sulfonated Peptides by a Water-Soluble Fullerene Derivative and its Applications to Highly Efficient Proteomics. Anal. Chim. Acta 2006, 556, 140-144. 
44. Leon, I. R.; Neves-Ferreira, A. G. C.; Valente, R. H.; Mota, E. M.; Lenzi, H. L.; Perales, J. Improved Protein Identification Efficiency by Mass Spectrometry Using N-Terminal Chemical Derivatization of Peptides from Angiostrongylus costaricensis, a Nematode with Unknown Genome. J. Mass Spectrom. 2007, 42, 1363-1374.

45. Wang, D.; Kalb Suzanne, R.; Cotter Robert, J. Improved Procedures for N-Terminal Sulfonation of Peptides for Matrix-Assisted Laser Desorption/Ionization Post-Source Decay Peptide Sequencing. Rapid Commun. Mass Spectrom. 2004, 18, 96-102.

46. Shin, J.-W: Lee, Y. H. Hwang, S. Lee, S.-W. Observation of an Unusually Facile Fragmentation Pathway of Gas-Phase Peptide Ions: A Study on the Gas-Phase Fragmentation Mechanism and Energetics of Tryptic Peptides Modified with 4-Sulfophenyl Isothiocyanate (SPITC) and 4-Chlorosulfophenyl Isocyanate (SPC) and Their 18-Crown-6 Complexes. J. Mass Spectrom. 2007, 42, 380-388.

47. Oh, J. Y.; Moon, J. H.; Lee, Y. H.; Hyung, S.-W.; Lee, S.-W.; Kim, M. S. Photodissociation Tandem Mass Spectrometry at $266 \mathrm{~nm}$ of an Aliphatic Peptide Derivatized with Phenyl Isothiocyanate and 4-Sulfophenyl Isothiocyanate. Rapid Commun. Mass Spectrom. 2005, 19, 1283-1288.

48. Gardner, M. W.; Vasicek, L. A.; Shabbir, S.; Anslyn, E. V.; Brodbelt, J. S. Chromogenic Cross-Linker for the Characterization of Protein Structure by Infrared Multiphoton Dissociation Mass Spectrometry. Anal. Chem. 2008, 80, 4807-4819.

49. Flora, J. W.; Muddiman, D. C. Selective, Sensitive, and Rapid Phosphopeptide Identification in Enzymatic Digests Using ESI-FTICR-MS with Infrared Multiphoton Dissociation. Anal. Chem. 2001, 73, 33053311.
50. Flora, J. W.; Muddiman, D. C. Gas-Phase Ion Unimolecular Dissociation for Rapid Phosphopeptide Mapping by IRMPD in a Penning Ion Trap: An Energetically Favored Process. J. Am. Chem. Soc. 2002, 124, 65466547

51. Flora, J. W.; Muddiman, D. C. Determination of the Relative Energies of Activation for the Dissociation of Aromatic vs. Aliphatic Phosphopeptides by ESI-FTICR-MS and IRMPD. J. Am. Soc. Mass Spectrom. 2004, 15, 121-127.

52. Jones, J. L.; Dongre, A. R.; Somogyi, A.; Wysocki, V. H. Sequence Dependence of Peptide Fragmentation Efficiency Curves Determined by Electrospray Ionization/Surface-Induced Dissociation Mass Spectrometry. J. Am. Chem. Soc. 1994, 116, 8368-8369.

53. Guillaume, E.; Panchaurd, A.; Affolter, M.; Desvergnes, V.; Kussmann, M. Differentially Isotope-Coded N-Terminal Protein Sulphonation: Combining Protein Identification and Quantification. Proteomics 2006, 6, $2338-2349$

54. Lee, Y. H.; Kim, M.-S.; Choie, W.-S.; Min, H.-K.; Lee, S.-W. Highly Informative Proteome Analysis by Combining Improved N-Terminal Sulfonation for De Novo Peptide Sequencing and On-Line Capillary Reverse-Phase Liquid Chromatography/Tandem Mass Spectrometry. Proteomics 2004, 4, 1684-1694.

55. Bunk, D. M.; Macfarlane, R. D. Derivatization to Enhance SequenceSpecific Fragmentation of Peptides and Proteins. Int. J. Mass Spectrom. Ion Processes 1993, 126, 123-136.

56. Beardsley, R. L.; Karty, J. A.; Reilly, J. P. Enhancing the Intensities of Lysine-Terminated Tryptic Peptide Ions in Matrix-Assisted Laser Desorption/Ionization Mass Spectrometry. Rapid Commun. Mass Spectrom. 2000, 14, 2147-2153. 